\title{
2016 International Society for Disease Surveillance Conference New Frontiers in Surveillance: Data Science and Health Security
}

The International Society for Disease Surveillance (ISDS) held its fifteenth annual conference in Atlanta, GA, from December 6-8, 2016. Since 2001, individuals interested in sharing and learning emerging trends in surveillance research and practice have found the ISDS Annual Conference a unique forum to advance their knowledge in the discipline of disease surveillance. The $15^{\text {th }}$ ISDS conference received a total of 233 abstracts from 23 countries. From the submissions, 189 $(81 \%)$ were accepted for presentation at the conference as an oral presentation $(\mathrm{N}=96)$ or poster $(\mathrm{N}=93)$.

The theme for the $15^{\text {th }}$ annual conference was New Frontiers in Surveillance: Data Science and Health Security. The theme united two dominant trends in public health surveillance: 1) a growing desire to extract knowledge from increasing volumes of structured and unstructured data available from health information systems; and 2) increased pressure on nations to strengthen their capacity for disease surveillance and response to outbreaks when and where they occur across the globe. In addition to the major themes of the conference, abstracts were accepted in additional tracks that remain important to the practice of public health around the world: One Health uniting animal and human health; Methodological advances in applied epidemiology; Public health informatics; Public health policy; and Biosurveillance practice.

As usual, accepted abstracts for the 2016 ISDS Conference span the breadth of surveillance practice around the globe. There are timely abstracts on the detection and response to vector-borne diseases such as Zika virus and chikungunya across the Americas, as well as abstracts on the surveillance of opioid abuse observed in many parts of the U.S. Other abstracts cover the surveillance of non-communicable diseases that are now the leading causes of death globally. Additionally, some abstracts focus on capacity building within low resource settings on multiple continents to enhance global health security. While other abstracts describe the impact of health information technology (or eHealth) policies on surveillance practice at local, national, or regional levels. And still other abstracts contain emerging, novel methods that advance our understanding of how to analyze "Big" data or reduce the messiness associated with realworld surveillance data. Together these abstracts represent the broad, diverse and interesting nature of surveillance practice. Furthermore, the abstracts represent important work being done in high income countries like the U.S., Canada and the U.K. as well as critical work being done in low-and-middle income nations such as Nigeria, Pakistan, and Sierra Leone.

I wish to thank the dedicated members of the Scientific Programming Committee (SPC) and ISDS staff who helped to manage the process of selecting this year's abstracts for presentation. These individuals are domain experts across the spectrum of tracks and themes represented in the program, and their service is much appreciated. The SPC helped to recruit dozens of public health researchers and practitioners who also spent time reviewing abstracts. I also thank these volunteers for contributing to the richness and diversity of this year's program. Finally, I wish to thank the Track Chairs who reviewed abstracts and recruited peers to perform reviews, and whom helped me organize presentations into meaningful sessions for the final conference program. Their names are listed in the proceedings to recognize their selfless service to ISDS and the field of public health surveillance.

I hope that these proceedings help to advance scientific understanding and the practice of surveillance in public health. Please use the knowledge herein to improve how you practice or evaluate surveillance in your jurisdiction. Or you may find ways to apply the knowledge elsewhere in population health. However you use it, I ask that you document your lessons or findings and submit to ISDS in the future to share the outcomes with others. Together we can reduce the burden of disease and improve health outcomes for populations globally.

Brian E. Dixon, MPA, PhD, FHIMSS

Indiana University and the Regenstrief Institute, Inc. 2016 ISDS Scientific Program Committee Chair

Track Chairs for the 2016 ISDS Conference

Eric Lau, PhD

School of Public Health, the University of Hong Kong Chair for Methodologies and Analytics

Julie Pavlin, MD, PhD, MPH Infectious Disease Clinical Research Program Chair for Public Health Policy

Peter Hicks, MA, MPH

Centers for Disease Control and Prevention $(C D C)$ Chair for Informatics and Data Science

Vivek Singh, MPH, MBBS

Indian Institute of Public Health (IIPH) - Hyderabad Chair for Public/Population Health Practice 